\title{
GROUPS WITH A NONTRIVIAL NONIDEAL KERNEL
}

\author{
FRIEDER LADISCH
}

\begin{abstract}
A Bs TRACT. We classify finite groups $G$, such that the group algebra, $\mathbb{Q} G$ (over the field of rational numbers $\mathbb{Q}$ ), is the direct product of the group algebra $\mathbb{Q}[G / N]$ of a proper factor group $G / N$, and some division rings.
\end{abstract}

\section{INTRODUCTION}

Let $G$ be a finite group and $\mathbb{K}$ a field of characteristic zero. By Maschke's theorem and Wedderburn-Artin theory, the group algebra $\mathbb{K} G$ of $G$ over $\mathbb{K}$ is a direct product of matrix rings over division algebras:

$$
\mathbb{K} G \cong \mathbf{M}_{d_{1}}\left(D_{1}\right) \times \cdots \times \mathbf{M}_{d_{r}}\left(D_{r}\right) .
$$

A natural question to ask is when each factor in this decomposition is actually a division ring (equivalently, the group algebra $\mathbb{K} G$ contains no nilpotent elements). In the classical case where $\mathbb{K}$ is algebraically closed, it is well known that $\mathbb{K} G$ is a direct product of division rings if and only if $G$ is abelian. For $\mathbb{K}=\mathbb{Q}$, the question was solved by S. K. Seghal [14, Theorem 3.5] (see Theorem 4.5 below).

In this paper, we consider a slightly more general question: Let $1 \neq N \unlhd G$ be a normal subgroup. Then

$$
\mathbb{K} G \cong \mathbb{K}[G / N] \times I,
$$

where the (twosided) ideal $I$ is the kernel of the canonical homomorphism $\mathbb{K} G \rightarrow$ $\mathbb{K}[G / N]$. Now we ask: for which finite groups is there an $N \neq 1$ such that the ideal $I$ above is a direct product of division rings? If there is such an $N$, then any nilpotent element of $\mathbb{K} G$ has constant coefficients on cosets of $N$. Also, only twosided ideals of $\mathbb{K} G$ can distinguish the elements of $N$.

The following is just a basic observation, which allows us to state our results more conveniently.

Lemma A. For each field $\mathbb{K}$ (of characteristic zero) and each finite group $G$, there is a unique maximal normal subgroup $N$, denoted by $\operatorname{NKer}_{\mathbb{K}}(G)$, such that the kernel of the map $\mathbb{K} G \rightarrow \mathbb{K}[G / N]$ is a direct product of division rings.

2010 Mathematics Subject Classification. 20C15.

Key words and phrases. Characters, Finite groups, Representations, Schur indices, Division rings.

Author supported by the DFG (Project: SCHU 1503/6-1). 
We will give a more direct definition of $\operatorname{NKer}_{\mathbb{K}}(G)$ in Section 3 below, before we prove Lemma A. We call $\operatorname{NKer}_{\mathbb{K}}(G)$ the nonideal kernel of $G$ (over $\mathbb{K}$ ).

We view the zero ideal as an empty product of division rings, so possibly $\mathrm{NKer}_{\mathbb{K}}(G)=1$. Indeed, this is the case for "most" groups, and we want to classify the groups $G$ for which $\operatorname{NKer}_{\mathbb{K}}(G) \neq 1$. Our first result concerns the field $\mathbb{R}$ of real numbers.

We need to recall a definition: A nonabelian group $G$ is called generalized dicyclic, if it has an abelian subgroup $A$ of index 2 and an element $g \in G \backslash A$ such that $g^{2} \neq 1$ and $a^{g}=a^{-1}$ for all $a \in A$. If $A$ is cyclic, then $G$ is called dicyclic (or generalized quaternion). Furthermore, $Q_{8}$ denotes the quaternion group of order 8 and $C_{n}$ a cyclic group of order $n$.

Theorem B. Let $G$ be a finite group. Then $\operatorname{NKer}_{\mathbb{R}}(G)>1$ if and only if one of the following holds:

(i) $G$ is abelian and $G \neq\{1\}$.

(ii) $G$ is generalized dicyclic.

(iii) $G \cong C_{4} \times Q_{8} \times\left(C_{2}\right)^{r}, r \in \mathbb{N}$.

(iv) $G \cong Q_{8} \times Q_{8} \times\left(C_{2}\right)^{r}, r \in \mathbb{N}$.

The motivation for this work is a question of Babai [1]. Babai asked which finite groups are isomorphic to the affine symmetry group of an orbit polytope. (An orbit polytope is a polytope such that its (affine) symmetry groups acts transitively on the vertices of the polytope.) In joint work with Erik Friese [7] (continuing our earlier paper [6]), we develop a general theory, which shows, among other things, that $G$ is isomorphic to the affine symmetry group of an orbit polytope when $\operatorname{NKer}_{\mathbb{R}}(G)=1$. When $\operatorname{NKer}_{\mathbb{R}}(G)>1$, this may or may not be the case. Theorem B above is an essential ingredient in our answer to Babai's question. Similarly, when $\operatorname{NKer}_{\mathbb{Q}}(G)=1$, then $G$ can be realized as the affine symmetry group of an orbit polytope with vertices having rational coordinates.

The classification of groups with $\operatorname{NKer}_{\mathbb{Q}}(G)>1$ is more complicated. To state it, we first describe a special type of such groups.

Lemma C. Let $p \neq q$ be primes, let $P=\langle g\rangle \times P_{0}$ be an abelian $p$-group and $Q$ an abelian q-group. Suppose $P$ acts on $Q$ such that $x^{g}=x^{k}$ for all $x \in Q$ and some integer $k$ independent of $x \in Q$, and such that $\mathbf{C}_{P}(Q)=\left\langle g^{p^{c}}\right\rangle \times P_{0}$ for some $c \geqslant 1$. Suppose that $p^{d}=\operatorname{ord}\left(g^{p^{c}}\right)$ is the exponent of $\mathbf{C}_{P}(Q)$, and that $(q-1)_{p}$, the p-part of $q-1$, divides $p^{d}$. If $p=(q-1)_{p}=2$, assume additionally that $\left(q^{2}-1\right)_{2}$ divides $2^{d}$. Then for the semidirect product $G=P Q$, we have $\operatorname{NKer}_{\mathbb{Q}}(G) \cap\langle g\rangle \neq 1$.

Notice that the assumption on the action of $g$ on $Q$ and $\left|P / \mathbf{C}_{P}(Q)\right|=p^{c}$ imply that $p^{c}$ divides $q-1$, and that the multiplicative order of $k$ modulo the exponent of $Q$ is just $p^{c}$. As we will see later, $1 \neq \operatorname{NKer}_{\mathbb{Q}}(G) \leqslant\left\langle g^{p^{c}}\right\rangle$. Whenever we mention "groups as in Lemma C", we also use the notation established in the statement of Lemma C. 
Theorem D. Let $G$ be a finite group. Then $\operatorname{NKer}_{\mathbb{Q}}(G) \neq 1$ if and only if at least one of the following holds:

(i) $G$ is abelian and $G \neq\{1\}$.

(ii) $G=S \times A$, where $S$ is a 2-group of exponent 4 which appears on the list from Theorem $B$, the group $A$ is abelian of odd order, and the multiplicative order of 2 modulo $|A|$ is odd.

(iii) $G$ is generalized dicyclic.

(iv) $G=(P Q) \times B$, where the subgroups $P \in \operatorname{Syl}_{p}(G), Q \in \operatorname{Syl}_{q}(G)$ and $B$ are abelian, $P Q$ is as in Lemma $C$, and the p-part of the multiplicative order of $q$ modulo $|B|$ divides the multiplicative order of $q$ modulo $p^{d}$.

(v) $G=Q_{8} \times\left(C_{2}\right)^{r} \times H$, where $H$ is as in (iv) and has odd order, and the multiplicative order of 2 modulo $|H|$ is odd.

Case (ii) contains the groups $G=Q_{8} \times\left(C_{2}\right)^{r} \times A$, for which $\mathbb{Q} G$ is a direct product of division rings, as classified by Sehgal [14].

An important tool in the proofs of Theorems B and D is Blackburn's classification of finite groups in which all nonnormal subgroups have a nontrivial intersection [3]. As we will see below, $\operatorname{NKer}_{\mathbb{K}}(G)$ is always contained in the intersection of all nonnormal subgroups of $G$. While the proof of Theorem B is relatively elementary, the proof of Theorem $\mathrm{D}$ also depends on some deep facts about division algebras and Schur indices. Recently, Erik Friese found a proof of Theorem B which does not depend on Blackburn's classification [5, Theorem 6.2.3].

The paper is organized as follows: In Section 2, we review some basic facts about representations and characters over fields not necessarily algebraically closed, and in particular Schur indices. We also introduce the auxiliary concept of skew-linear characters. In Section 3, we define $\operatorname{NKer}_{\mathbb{K}}(G)$ and prove some elementary properties. In Section 4, we consider Dedekind groups (groups such that all subgroups are normal). In such groups, we have either $\operatorname{NKer}_{\mathbb{K}}(G)=1$, or $\operatorname{NKer}_{\mathbb{K}}(G)=G$, where the latter are exactly the groups such that $\mathbb{K} G$ is a direct product of division rings. Finally, Section 5 contains the proof of Theorem B, and Section 6 the (long) proof of Theorem D.

\section{SKEW-LINEAR CHARACTERS}

Let $G$ be a finite group. For simplicity, assume that $\mathbb{K} \subseteq \mathbb{C}$ and write $\operatorname{Irr} G$ for the set of irreducible complex characters of $G$. We begin by reviewing the relation between the representation theory of $G$ over $\mathbb{K}$ and over $\mathbb{C}[9, \S 38][10$, Chapter 10].

By Maschke's theorem and general Wedderburn-Artin theory, the group algebra $\mathbb{K} G$ is the direct product of simple rings:

$$
\mathbb{K} G=A_{1} \times \cdots \times A_{r} .
$$


Each $A_{i}$ is a simple ideal, and the set of the $A_{i}$ 's is uniquely determined as the set of simple ideals of $\mathbb{K} G$. The $A_{i}$ 's are called the block ideals of $\mathbb{K} G$. Each $A_{i}$ is generated by a centrally primitive idempotent $e \in \mathbf{Z}(\mathbb{K} G)$. By Wedderburn-Artin theory, each $A_{i}$ is isomorphic to a matrix ring over a division ring.

We now relate the above decomposition to the complex irreducible characters of $G$. Recall that the Schur index of $\chi \in \operatorname{Irr} G$ over $\mathbb{K}$ is the smallest positive integer $m=m_{\mathbb{K}}(\chi)$ such that $m \chi$ is afforded by a representation with entries in $\mathbb{K}(\chi)$, the field generated by $\mathbb{K}$ and the values of $\chi$.

2.1. Lemma. Let $\chi \in \operatorname{Irr} G$.

(i) There is a unique block ideal $A=A_{\mathbb{K}}(\chi)$ of $\mathbb{K} G$ such that $\chi(A) \neq 0$.

(ii) Let $\psi \in \operatorname{Irr} G$. Then $\psi(A) \neq 0$ if and only if $\psi$ and $\chi$ are Galois conjugate over $\mathbb{K}$, that is, $\psi=\chi^{\alpha}$ for some $\alpha \in \operatorname{Gal}(\mathbb{K}(\chi) / \mathbb{K})$.

(iii) Write $A \cong \mathbf{M}_{n}(D)$ for some division ring $D=D_{\mathbb{K}}(\chi)$. Then $\mathbf{Z}(A) \cong$ $\mathbf{Z}(D) \cong \mathbb{K}(\chi)$.

(iv) $|D: \mathbf{Z}(D)|=m_{\mathbb{K}}(\chi)^{2}$ and $\chi(1)=n m_{\mathbb{K}}(\chi)$.

Proof. This is standard [9, Theorems 38.1 and 38.15].

It follows from Lemma 2.1 that $A=A_{\mathbb{K}}(\chi)$ is itself a division ring if and only if $\chi(1)=m_{\mathbb{K}}(\chi)$. In this case, the projection $\mathbb{K} G \rightarrow A$ defines a homomorphism $\varphi$ from $G$ into the multiplicative group of $D$. Notice also that $\operatorname{Ker}(\varphi)=\operatorname{Ker}(\chi)$. For this reason, we call a character $\chi$ skew-linear (over $\mathbb{K})$, if $\chi(1)=m_{\mathbb{K}}(\chi)$. Thus skew-linear characters generalize linear characters. Since $m_{\mathbb{C}}(\chi)=1$ for all $\chi$, skew-linear over $\mathbb{C}$ is the same as linear.

If $\chi \in \operatorname{Irr}(G)$ is linear, then (trivially) the reduction to any subgroup is irreducible and linear. This fact generalizes to skew-linear characters as follows:

2.2. Lemma. Let $\chi \in \operatorname{Irr}(G)$ be skew-linear over the field $\mathbb{K}$, and $H \leqslant G$. Then the irreducible constituents of $\chi_{H}$ are skew-linear over $\mathbb{K}$, and are Galois conjugate over the field $\mathbb{K}(\chi)$.

Proof. Let $\vartheta \in \operatorname{Irr}(H)$ be a constituent of $\chi_{H}$. Then [10, Lemma 10.4]

$$
m_{\mathbb{K}}(\chi) \text { divides }\left[\chi_{H}, \vartheta\right] \cdot|\mathbb{K}(\chi, \vartheta): \mathbb{K}(\chi)| \cdot m_{\mathbb{K}}(\vartheta) .
$$

Let $\sigma \in \operatorname{Gal}(\mathbb{K}(\chi, \vartheta) / \mathbb{K}(\chi))$. Then $\left[\chi_{H}, \vartheta^{\sigma}\right]=\left[\chi_{H}, \vartheta\right]$. Thus each of the $\mid \mathbb{K}(\chi, \vartheta)$ : $\mathbb{K}(\chi) \mid$ characters $\vartheta^{\sigma}$ occurs in $\chi_{H}$ with multiplicity $\left[\chi_{H}, \vartheta\right]$. It follows that

$$
\begin{aligned}
{\left[\chi_{H}, \vartheta\right] \cdot|\mathbb{K}(\chi, \vartheta): \mathbb{K}(\chi)| \cdot \vartheta(1) } & \leqslant \chi(1)=m_{\mathbb{K}}(\chi) \\
& \leqslant\left[\chi_{H}, \vartheta\right] \cdot|\mathbb{K}(\chi, \vartheta): \mathbb{K}(\chi)| \cdot m_{\mathbb{K}}(\vartheta) \\
& \leqslant\left[\chi_{H}, \vartheta\right] \cdot|\mathbb{K}(\chi, \vartheta): \mathbb{K}(\chi)| \cdot \vartheta(1) .
\end{aligned}
$$

This implies that equality holds throughout, in particular, $\vartheta(1)=m_{\mathbb{K}}(\vartheta)$ and $\chi_{H}=\left[\chi_{H}, \vartheta\right] \sum \vartheta^{\sigma}$, the sum running over $\sigma \in \operatorname{Gal}(\mathbb{K}(\chi, \vartheta) / \mathbb{K}(\chi))$. 
In the rest of this section, we record some (mostly well known) facts about Schur indices and blocks of group algebras for later reference.

Recall that

$$
e_{\chi}=\frac{\chi(1)}{|G|} \sum_{g \in G} \chi\left(g^{-1}\right) g
$$

is the centrally primitive idempotent in $\mathbb{C} G$ corresponding to $\chi \in \operatorname{Irr} G$. The following simple observation will sometimes be useful. Notice that it provides an alternative proof of $\mathbf{Z}(A) \cong \mathbb{K}(\chi)$.

2.3. Lemma. Let $\chi \in \operatorname{Irr} G$ and let $A$ be the block ideal of $\mathbb{K} G$ such that $\chi(A) \neq 0$. Then

$$
A \cong \mathbb{K}(\chi) G e_{\chi} \quad \text { by } \quad A \ni a \mapsto a e_{\chi}
$$

Proof. Set

$$
e:=\sum_{\alpha \in \operatorname{Gal}(\mathbb{K}(\chi) / \mathbb{K})} e_{\chi^{\alpha}}
$$

We claim that $A=\mathbb{K} G e$. We can decompose 1 into a sum of primitive idempotents in $\mathbf{Z}(\mathbb{K} G)$, and then decompose further in $\mathbf{Z}(\mathbb{C} G)$. Thus there is a unique primitive idempotent $f$ in $\mathbf{Z}(\mathbb{K} G)$ such that $f e_{\chi}=e_{\chi}$. But then also $f e_{\chi^{\alpha}}=e_{\chi^{\alpha}}$ for all $\alpha \in \operatorname{Gal}(\mathbb{K}(\chi) / \mathbb{K})$ and thus $f e=e$. On the other hand, $e_{\chi} \in \mathbb{K}(\chi) G$ and $e \in \mathbb{K} G$, and thus $f=e$. This shows that $A=\mathbb{K} G e$ as claimed.

For $\alpha \in \operatorname{Gal}(\mathbb{K}(\chi) / \mathbb{K})$,

$$
b=\sum_{g} b_{g} g \in \mathbb{K}(\chi) G e_{\chi} \quad \text { implies } \quad b^{\alpha}:=\sum_{g} b_{g}^{\alpha} g \in \mathbb{K}(\chi) G e_{\chi^{\alpha}} .
$$

Using this, it is straightforward to check that

$$
\mathbb{K}(\chi) G e_{\chi} \ni b \mapsto \sum_{\alpha \in \operatorname{Gal}(\mathbb{K}(\chi) / \mathbb{K})} b^{\alpha} \in \mathbb{K} G e
$$

yields the inverse of the map $a \mapsto a e_{\chi}$.

Since we will often have to consider characters of direct products of groups, and the corresponding blocks of the group algebra, we record the following for later reference.

2.4. Lemma. Let $G=U \times V$ be a direct product of groups, $\sigma \in \operatorname{Irr} U$ and $\tau \in \operatorname{Irr} V$. Then $\chi=\sigma \times \tau \in \operatorname{Irr} G$ and $\mathbb{K}(\chi)=\mathbb{K}(\sigma, \tau)$. Let $A_{\mathbb{K}}(\chi)$ be the block ideal of $\mathbb{K} G$ corresponding to $\chi$, and $A_{\mathbb{K}}(\sigma)$ and $A_{\mathbb{K}}(\tau)$ the block ideals of $\mathbb{K} U$ and $\mathbb{K} V$ corresponding to $\sigma$ and $\tau$. Then

$$
A_{\mathbb{K}}(\chi) \cong\left(A_{\mathbb{K}}(\sigma) \otimes_{\mathbb{K}(\sigma)} \mathbb{K}(\chi)\right) \otimes_{\mathbb{K}(\chi)}\left(A_{\mathbb{K}}(\tau) \otimes_{\mathbb{K}(\tau)} \mathbb{K}(\chi)\right) .
$$

Proof. The irreducible characters of $U \times V$ are exactly the characters of the form $\chi=\sigma \times \tau$, with $\sigma \in \operatorname{Irr} U$ and $\tau \in \operatorname{Irr} V\left[10\right.$, Theorem 4.21]. Since $\chi\left(\left(u, 1_{V}\right)\right)=$ $\sigma(u) \tau\left(1_{V}\right)$ for $u \in U$ and similarly $\chi\left(\left(1_{U}, v\right)\right)=\sigma\left(1_{U}\right) \tau(v)$ for $v \in V$, we see that $\mathbb{K}(\chi)=\mathbb{K}(\sigma, \tau)$. 
Set $\mathbb{L}=\mathbb{K}(\chi)$. The natural isomorphism

$$
\mathbb{L} U \otimes_{\mathbb{L}} \mathbb{L} V \rightarrow \mathbb{L} G, \quad \sum_{u} a_{u} u \otimes \sum_{v} b_{u} v \mapsto \sum_{u, v} a_{u} b_{v}(u, v)
$$

sends $e_{\sigma} \otimes e_{\tau}$ to $e_{\chi}$ and thus induces an isomorphism

$$
\mathbb{L} U e_{\sigma} \otimes_{\mathbb{L}} \mathbb{L} V e_{\tau} \cong \mathbb{L} G e_{\chi}
$$

(by comparing dimensions). By Lemma 2.3, the right hand side is isomorphic to $A_{\mathbb{K}}(\chi)$, and on the left hand side we have

$$
\mathbb{L} U e_{\sigma} \cong \mathbb{K}(\sigma) U e_{\sigma} \otimes_{\mathbb{K}(\sigma)} \mathbb{L} \cong A_{\mathbb{K}}(\sigma) \otimes_{\mathbb{K}(\sigma)} \mathbb{L},
$$

and similarly for the other factor. The result follows.

In Section 6, we need several deep facts about Schur indices, which we collect now. For a prime $q$, we write $m_{q}(\chi):=m_{\mathbb{Q}_{q}}(\chi)$, where $\mathbb{Q}_{q}$ denotes the field of $q$-adic numbers. Sometimes, it will be convenient to use this notation also for the "infinite prime", that is, $m_{\infty}(\chi):=m_{\mathbb{R}}(\chi)$.

2.5. Lemma. Let $\chi \in \operatorname{Irr}(G)$.

(i) $m_{\mathbb{Q}}(\chi)$ is the least common multiple of the local indices $m_{q}(\chi)$, where $q$ runs through all primes, including the infinite one. [13, (32.19)]

(ii) $m_{\mathbb{R}}(\chi)$ and $m_{2}(\chi)$ divide 2 , and $m_{q}(\chi)$ divides $q-1$ for odd $q$. [16, Theorem 4.3, Corollary 5.5]

(iii) Let $\varphi$ be an irreducible Brauer character for the prime $q$, and $d_{\chi \varphi}$ the decomposition number. Then $m_{q}(\chi)$ divides $d_{\chi \varphi}\left|\mathbb{Q}_{q}(\chi, \varphi): \mathbb{Q}_{q}(\chi)\right|$. [4, Theorem IV.9.3]

(iv) If the finite prime $q$ does not divide $|G|$, then $m_{q}(\chi)=1$. [4, Corollary IV.9.5]

2.6. Corollary. Let $\chi \in \operatorname{Irr}(G)$ with $\chi(1)=m_{q}(\chi)$, where $q$ is a prime number. If $H \leqslant G$ has order not divisible by $q$, then any constituent of $\chi_{H}$ is linear.

Proof. This is immediate from Lemma 2.2 and Lemma 2.5 (iv).

\section{THE NONIDEAL KERNEL}

For every field $\mathbb{K}$ and any finite group $G$, we define

$$
\operatorname{NKer}_{\mathbb{K}}(G):=\bigcap\left\{\operatorname{Ker}(\chi) \mid \chi \in \operatorname{Irr}(G), \chi(1)>m_{\mathbb{K}}(\chi)\right\} .
$$

If $m_{\mathbb{K}}(\chi)=\chi(1)$ for every $\chi \in \operatorname{Irr}(G)$, we set $\operatorname{NKer}_{\mathbb{K}}(G):=G$. We call $\operatorname{NKer}_{\mathbb{K}}(G)$ the nonideal kernel of $G$ over $\mathbb{K}$. Notice that $\operatorname{NKer}_{\mathbb{K}}(G)$, for any field $\mathbb{K}$, is characteristic in $G$.

3.1. Lemma. Let $\mathbb{K} \subseteq \mathbb{L}$ be fields. Then $\operatorname{NKer}_{\mathbb{L}}(G) \subseteq \operatorname{NKer}_{\mathbb{K}}(G)$.

Proof. Since $m_{\mathbb{L}}(\chi)$ divides $m_{\mathbb{K}}(\chi)$ for any $\chi \in \operatorname{Irr} G$, any character which is skew-linear over $\mathbb{L}$, is also skew-linear over $\mathbb{K}$. The result follows. 
3.2. Lemma. Let $G$ be a nonabelian group. Then

$$
\bigcap_{\substack{\chi \in \operatorname{Irr} G \\ \chi(1)>1}} \operatorname{Ker} \chi=\{1\} .
$$

Proof. Suppose that $g \neq 1$ is contained in the kernel of all nonlinear characters. Then, by the second orthogonality relation [10, (2.18)],

$$
0=\sum_{\chi \in \operatorname{Irr} G} \chi(1) \chi(g)=\sum_{\substack{\chi \in \operatorname{Irr} G \\ \chi(1)>1}} \chi(1)^{2}+\sum_{\chi \in \operatorname{Lin} G} \chi(1) \chi(g) .
$$

The second sum runs over the irreducible characters of $G / G^{\prime}$ and has value $\left|G: G^{\prime}\right|$ or 0 , according to whether $g \in G^{\prime}$ or not. It follows that the first sum must be empty. Thus $G$ has no nonlinear characters, which means that $G$ is abelian, as claimed.

3.3. Corollary. Let $G$ be a nonabelian group. Then $\operatorname{NKer}_{\mathbb{C}}(G)=1$.

Let us say that a character $\alpha$ (not necessarily irreducible) is strictly nonideal, if $[\alpha, \chi]<\chi(1)$ for all $\chi \in \operatorname{Irr} G$. (Such a character is afforded by a left ideal of the group algebra, which does not contain any nonzero two-sided ideal.) If at the same time, $\alpha$ is the character of a representation with entries in $\mathbb{K}$, then $m_{\mathbb{K}}(\chi)$ divides $[\alpha, \chi]$ for all $\chi \in \operatorname{Irr} G$ [10, Corollary 10.2(c)]. Thus no constituent of $\alpha$ can be skewlinear over $\mathbb{K}$. Conversely, if $S$ is a set of non-skew-linear characters over $\mathbb{K}$, then we may add the characters of the corresponding irreducible representations over $\mathbb{K}$ and get a strictly nonideal character $\alpha$ which is afforded by a $\mathbb{K}$-representation. Since Ker $\alpha=\bigcap$ Ker $\chi$, where $\chi$ runs through the constituents of $\alpha$, it follows that every group $G$ has a strictly nonideal character $\alpha$ with $\operatorname{Ker} \alpha=\operatorname{NKer}_{\mathbb{K}}(G)$, and such that $\alpha$ is afforded by a representation over $\mathbb{K}$. (In the case where $G=\operatorname{NKer}_{\mathbb{K}}(G)$, the only such character is $\alpha=0$, however.)

3.4. Lemma. Let $H \leqslant G$ with $N:=\operatorname{NKer}_{\mathbb{K}}(H)<H$. Then

$$
\operatorname{NKer}_{\mathbb{K}}(G) \leqslant \bigcap_{g \in G} N^{g} \leqslant \operatorname{NKer}_{\mathbb{K}}(H)
$$

Proof. Let $\alpha$ be a strictly nonideal character of $H$ with $N=\operatorname{Ker} \alpha$ and which is afforded by a representation over $\mathbb{K}$. Then $0 \neq \alpha^{G}$ is afforded by a representation over $\mathbb{K}$ and has kernel $\bigcap_{g \in G} N^{g}$ [10, Lemma 5.11].

Let $\rho_{G}$ be the regular character of $G$. Notice that a character $\beta$ is strictly nonideal if and only if $\rho_{G}-\beta$ is a character and $\left[\rho_{G}-\beta, \chi\right]>0$ for all $\chi \in \operatorname{Irr} G$. Since $\rho_{G}=\left(\rho_{H}\right)^{G}$, we have that $\rho_{G}-\alpha^{G}=\left(\rho_{H}-\alpha\right)^{G}$ is a character, and

$$
\left[\rho_{G}-\alpha^{G}, \chi\right]=\left[\left(\rho_{H}-\alpha\right)^{G}, \chi\right]=\left[\rho_{H}-\alpha, \chi_{H}\right]_{H}>0
$$

for all $\chi \in \operatorname{Irr} G$. Thus $\alpha^{G}$ is strictly nonideal. 
3.5. Lemma. Let $N$ be a normal subgroup of $G$, and set

$$
e_{N}=\frac{1}{|N|} \sum_{n \in N} n
$$

Then $\mathbb{K} G e_{N} \cong \mathbb{K}[G / N]$. If $\chi \in \operatorname{Irr} G$, then $\chi\left(e_{N}\right) \neq 0$ if and only if $N \leqslant \operatorname{Ker}(\chi)$.

Proof. This is well known: The canonical epimorphism $\mathbb{K} G \rightarrow \mathbb{K}[G / N]$ is split by the map sending a coset $N g$ to $(1 /|N|) \sum N g=e_{N} g$. This proves the first statement.

If $N \leqslant \operatorname{Ker}(\chi)$, then any representation affording $\chi$ sends $e_{N}$ to the identity map. If $N \& \operatorname{Ker}(\chi)$, then any representation affording $\chi$ must send $e_{N}$ to 0 .

3.6. Lemma. Let $N:=\operatorname{NKer}_{\mathbb{K}}(G)$, and $e_{N}$ as in Lemma 3.5. Then $\mathbb{K} G\left(1-e_{N}\right)$ is a direct product of division rings. In particular, every idempotent $f \in \mathbb{K} G$ with $f e_{N}=0$ is central.

Proof. By Lemma 3.5, it follows that $\mathbb{K} G\left(1-e_{N}\right)$ is the direct product of the block ideals which correspond to $\chi \in \operatorname{Irr} G$ with $N \& \operatorname{Ker}(\chi)$. By definition of $N$, any such $\chi$ is skew-linear over $\mathbb{K}$, and thus the corresponding block ideal is a division ring.

In a direct product of division rings, every idempotent is central.

Proof of Lemma A. The first part of Lemma A is contained in Lemma 3.6. Conversely, if $\mathbb{K} G\left(1-e_{N}\right)$ is a direct product of division rings, then the above considerations yield that when $m_{\mathbb{K}}(\chi)<\chi(1)$, we must have $N \subseteq \operatorname{Ker}(\chi)$, and thus $N \leqslant \operatorname{NKer}_{\mathbb{K}}(G)$.

Following Blackburn [3], for any group $G$, we set

$$
\mathbf{R}(G):=\bigcap\{U \leqslant G \mid U \text { not normal in } G\} .
$$

If every subgroup of $G$ is normal, then we set $\mathbf{R}(G)=G$. Blackburn [3] classified finite groups in which $\mathbf{R}(G) \neq 1$. Therefore, a group $G$ with $\mathbf{R}(G) \neq 1$ is called a Blackburn group. The following result shows why this is relevant for us:

3.7. Lemma. For any finite group $G$ and field $\mathbb{K}$ of characteristic zero, we have $\operatorname{NKer}_{\mathbb{K}}(G) \leqslant \mathbf{R}(G)$.

Proof. Suppose $U \leqslant G$ is such that $N:=\operatorname{NKer}_{\mathbb{K}}(G) \nsubseteq \amalg \leqslant G$. We need to show that $U \unlhd G$.

Set $f=\left(1-e_{N}\right) e_{U}$, with $e_{N}$ as above, and $e_{U}:=(1 /|U|) \sum_{u \in U} u$ analogously. Then $f^{2}=f \in \mathbb{K} G\left(1-e_{N}\right)$, since $e_{N}$ is central in $\mathbb{K} G$. Thus $f$ is central in $\mathbb{K} G$ by Lemma 3.6. We compute

$$
f=\frac{1}{|U|} \sum_{u \in U} u-\frac{1}{|N U|} \sum_{x \in N U} x
$$

As $N$ is not contained in $U$, we have $U<N U$. As $g^{-1} f g=f$ for all $g \in G$, it follows that $U \unlhd G$. 


\section{DEDEKIND GROUPS}

In this section, we compute $\operatorname{NKer}_{\mathbb{K}}(G)$ for Dedekind groups, and determine when $\mathbb{K} G$ is a direct product of division rings. These results are mostly known.

Recall that a Dedekind group is a finite group in which all subgroups are normal. First, we recall Dedekind's classification of these groups [8, Satz III.7.12 on p. 308].

4.1. Theorem (Dedekind 1897). Let $G$ be a finite group, such that every subgroup of $G$ is normal. Then either $G$ is abelian, or

$$
G \cong Q_{8} \times\left(C_{2}\right)^{r} \times A \quad(r \geqslant 0),
$$

where $A$ is abelian of odd order.

Let $\tau \in \operatorname{Irr}\left(Q_{8}\right)$ be the irreducible, faithful character of degree 2 . Then $\mathbb{H}:=$ $\mathbb{Q} Q_{8} e_{\tau}$ is a division ring, the rational quaternions. $\mathbb{H}$ can also be described as the $\mathbb{Q}$-vector space with basis $\{1, i, j, k\}$ and multiplication defined by $i^{2}=j^{2}=-1$, $k=i j=-j i$.

4.2. Theorem. Let $\mathbb{K}$ be a field and $G$ be a group. Then $\mathbb{K} G$ is a direct product of division rings if and only if either $G$ is abelian, or $G \cong Q_{8} \times\left(C_{2}\right)^{r} \times A$, where $A$ is abelian of odd order, and $\mathbb{H} \otimes_{\mathbb{Q}} \mathbb{K}(\lambda)$ is a division ring for all $\lambda \in \operatorname{Lin}(A)$.

Proof. Suppose $\mathbb{K} G$ is a direct product of division rings. Then all subgroups of $G$ are normal in $G$ by Lemma 3.7 (as $\operatorname{NKer}_{\mathbb{K}}(G)=G$, or directly from the argument in the proof of Lemma 3.7). It follows that either $G$ is abelian, or $G \cong Q_{8} \times\left(C_{2}\right)^{r} \times A$ with $A$ abelian of odd order.

In the second case, let $\tau \in \operatorname{Irr}\left(Q_{8}\right)$ be the irreducible, faithful character of degree 2. Then

$$
\mathbb{K} Q_{8} e_{\tau} \cong \mathbb{H} \otimes_{\mathbb{Q}} \mathbb{K},
$$

the quaternions over $\mathbb{K}$. Any nonlinear, irreducible character of $G=Q_{8} \times\left(C_{2}\right)^{r} \times A$ has the form $\chi=\tau \times \sigma \times \lambda$, where $\sigma \in \operatorname{Lin}\left(C_{2}\right)^{r}$ and $\lambda \in \operatorname{Lin} A$. The corresponding block ideal of the rational group algebra is, by Lemma 2.4, isomorphic to

$$
\mathbb{H} \otimes_{\mathbb{Q}} \mathbb{K}(\lambda) .
$$

The result follows.

4.3. Corollary. Let $G$ be a Dedekind group and $\mathbb{K}$ a field. Then $\operatorname{NKer}_{\mathbb{K}}(G)=G$ or $\operatorname{NKer}_{\mathbb{K}}(G)=1$.

Proof. Suppose that $\operatorname{NKer}_{\mathbb{K}}(G) \neq G$, which means that $\mathbb{K} G$ is not a direct product of division rings. Then $G$ is not abelian and thus $G \cong Q_{8} \times\left(C_{2}\right)^{r} \times A$ with $A$ abelian of odd order. Moreover, there is some $\lambda \in \operatorname{Lin}(A)$ such that $\mathbb{H} \otimes_{\mathbb{Q}} \mathbb{K}(\lambda)$ is not a division ring. As before, let $\tau \in \operatorname{Irr}\left(Q_{8}\right)$ be the faithful irreducible character of $Q_{8}$. Then $\operatorname{Ker}(\tau \times 1 \times \lambda)=1 \times\left(C_{2}\right)^{r} \times \operatorname{Ker}(\lambda)$. 
It follows that $\operatorname{NKer}_{\mathbb{K}}(G) \subseteq 1 \times \operatorname{Ker}(\mu)$ for every $\mu \in \operatorname{Lin}\left(\left(C_{2}\right)^{r} \times A\right)$ such that $\operatorname{ord}(\lambda)$ divides the order of $\mu$. Since $A$ contains elements of order $\operatorname{ord}(\lambda)$, we see that $\operatorname{NKer}_{\mathbb{K}}(G)=1$.

Notice that for a linear character $\lambda$, we have $\mathbb{K}(\lambda)=\mathbb{K}\left(\varepsilon_{n}\right)$, where $\varepsilon_{n}$ is a primitive $n$-th root of unity and $n=\operatorname{ord}(\lambda)$. The following lemma collects some results. These will be needed also in the proof of Theorem D.

\subsection{Lemma.}

(i) $\mathbb{H} \otimes_{\mathbb{Q}} \mathbb{K}$ is a division ring if and only if -1 is not a sum of two squares in $\mathbb{K}$.

(ii) $\mathbb{H} \otimes_{\mathbb{Q}} \mathbb{Q}_{2}$ and $\mathbb{H} \otimes_{\mathbb{Q}} \mathbb{R}$ are division rings, and $\mathbb{H} \otimes_{\mathbb{Q}} \mathbb{Q}_{p}$ for $p$ odd is not a division ring. (Here $\mathbb{Q}_{p}$ is the field of p-adic numbers.)

Let $\varepsilon_{n}$ be a primitive $n$-th root of unity, where $n$ is odd. Then

(iii) $\mathbb{H} \otimes_{\mathbb{Q}} \mathbb{Q}\left(\varepsilon_{n}\right)$ is a division ring if and only if the multiplicative order of 2 in $(\mathbb{Z} / n)^{*}$ is odd, if and only if $\mathbb{H} \otimes_{\mathbb{Q}} \mathbb{Q}_{2}\left(\varepsilon_{n}\right)$ is a division ring.

(iv) $\mathbb{H} \otimes_{\mathbb{Q}} \mathbb{Q}\left(\sqrt{2}, \varepsilon_{n}\right)$ is a division ring only when $n=1$.

Proof. (i) and (ii) are well known [9, Example 38.13(a)] [15, Ch. III, Théorème 1]. Assertion (iii) is a result of Moser [12]. (This can be shown without using the HasseMinkowski principle: If the residue class of 2 in $(\mathbb{Z} / p)^{*}$ has even multiplicative order $2 r$, then $2^{r} \equiv-1 \bmod p$, and thus $p$ divides $2^{r}+1$. Then an elementary argument shows that -1 is a sum of two squares in $\mathbb{Q}\left(\varepsilon_{p}\right)$ [9, Example 38.13(d)]. If 2 has odd order in $(\mathbb{Z} / n \mathbb{Z})$, then $\mathbb{H} \otimes_{\mathbb{Q}} \mathbb{Q}_{2}\left(\varepsilon_{n}\right)$ is also a division ring.)

To see (iv), assume that $n>1$. We have to show that -1 is a sum of two squares in $\mathbb{K}:=\mathbb{Q}\left(\sqrt{2}, \varepsilon_{n}\right)$. By the Hasse-Minkowski principle, it suffices to show that -1 is a square in each possible completion of $\mathbb{K}$. Since $n>1$, $\mathbb{K}$ can not be embedded into $\mathbb{R}$. If $p$ is odd, then -1 is a sum of two squares in $\mathbb{Q}_{p}$ already. Finally, $\mathbb{Q}_{2}(\sqrt{2})$ is a quadratic extension of $\mathbb{Q}_{2}$ and thus a splitting field of $\mathbb{H}[11$, Lemma VI.2.14]. (We notice that in (iv), we can replace $\mathbb{Q}(\sqrt{2})$ by any field such that the completions at all prime ideals over 2 yield extensions of even degree over $\mathbb{Q}_{2}$.)

As a consequence, we get the following results.

4.5. Theorem (Sehgal $1975[14]$ ). The group algebra $\mathbb{Q} G$ is a direct product of division rings if and only if one of the following holds:

(i) $G$ is abelian.

(ii) $G \cong Q_{8} \times\left(C_{2}\right)^{r} \times A$, where $r \geqslant 0$, and $A$ is abelian of odd order, and the multiplicative order of 2 in $(\mathbb{Z} /|A|)^{*}$ is odd.

\subsection{Theorem.}

(i) $\mathbb{Q}_{2} G$ is a direct product of division rings if and only if $\mathbb{Q} G$ is a direct product of division rings. 
(ii) Let $p$ be an odd prime. Then $\mathbb{Q}_{p} G$ is a direct product of division rings if and only if $G$ is abelian.

(iii) $\mathbb{R} G$ is a direct product of division rings if and only if either $G$ is abelian, or $G \cong Q_{8} \times\left(C_{2}\right)^{r}$ for some $r \geqslant 0$.

\section{Clasification OVER The REALS}

In this section, we prove Theorem B. We start with a simple observation.

5.1. Lemma. Suppose that $\operatorname{NKer}_{\mathbb{R}}(G) \neq 1$, and $\langle g\rangle \not G$. Then $g$ has order 4 , and $\operatorname{NKer}_{\mathbb{R}}(G)=\mathbf{R}(G)=\left\langle g^{2}\right\rangle$ has order 2 .

Proof. By Lemma 3.7 and the definition of $\mathbf{R}(G)$, we have

$$
1 \neq N:=\operatorname{NKer}_{\mathbb{R}}(G) \leqslant \mathbf{R}(G)<\langle g\rangle .
$$

The last inequality is strict since $\mathbf{R}(G)$ is normal in $G$, but $\langle g\rangle$ is not. In particular, the first claim of the lemma implies the second one.

Let $\lambda \in \operatorname{Lin}\langle g\rangle$ be faithful. By Lemma 3.5 applied to $N \unlhd\langle g\rangle$ and since $N \neq 1$, it follows $\lambda\left(e_{N}\right)=0$. Thus

$$
f=e_{\lambda}+e_{\bar{\lambda}}=\frac{1}{|\langle g\rangle|} \sum_{h \in\langle g\rangle}(\bar{\lambda}(h)+\lambda(h)) h \in \mathbb{R}\langle g\rangle
$$

is an idempotent with $f e_{N}=0$. It follows from Lemma 3.6 that $f$ is a central idempotent in $\mathbb{R} G$, and so $f^{x}=f$ for all $x \in G$. But by assumption, there is some $x \in G$ such that $g^{x} \notin\langle g\rangle$. It follows that $\overline{\lambda(g)}+\lambda(g)=0$. As $\lambda(g)$ is an $n$-th root of unity, where $n=\operatorname{ord}(g)$, this is only possible when $\operatorname{ord}(g)=4$.

By this lemma, $\left|\operatorname{NKer}_{\mathbb{R}}(G)\right| \leqslant 2$ except when $G$ is a Dedekind group. Next we want to compute $\operatorname{NKer}_{\mathbb{R}}(G)$ in each case of Theorem B. In particular, this will prove the "if" part of Theorem B.

To show that certain characters are skew-linear, we use the Frobenius-Schur indicator. Recall that for $\chi \in \operatorname{Irr} G$, its Frobenius-Schur indicator is defined by

$$
\nu_{2}(\chi):=\frac{1}{|G|} \sum_{g \in G} \chi\left(g^{2}\right) .
$$

When $\nu_{2}(\chi)=1$, then $\chi=\bar{\chi}$ and $\chi$ is afforded by a representation with entries in $\mathbb{R}$, so $m_{\mathbb{R}}(\chi)=1$. When $\nu_{2}(\chi)=0$, then $\chi \neq \bar{\chi}$, and again $m_{\mathbb{R}}(\chi)=1$. Finally, when $\nu_{2}(\chi)=-1$, then $\chi=\bar{\chi}$, but $m_{\mathbb{R}}(\chi)=2$. In the last case, there is a simple $\mathbb{R} G$-module affording $2 \chi$, and $\operatorname{End}_{\mathbb{R} G}(S) \cong \mathbb{H}$, the division ring of Hamilton's quaternions [9, Theorem 13.12].

In particular, $\chi \in \operatorname{Irr} G$ is skew-linear over $\mathbb{R}$, if and only if either $\chi(1)=1(\chi$ is linear), or $\chi(1)=2$ and $\nu_{2}(\chi)=-1$. 
When $G$ is abelian, then trivially $\operatorname{NKer}_{\mathbb{R}}(G)=G$. Next we consider generalized dicyclic groups. Notice that the Dedekind group $Q_{8} \times\left(C_{2}\right)^{r}$ is generalized dicyclic, with abelian subgroup $A \cong C_{4} \times\left(C_{2}\right)^{r}$.

5.2. Lemma. Let $G$ be generalized dicyclic, and let $g \in G$ and $A \unlhd G$ be as in the definition (before Theorem B). Then $\mathbf{R}(G)=\operatorname{NKer}_{\mathbb{R}}(G)=G$ if $G /\left\langle g^{2}\right\rangle$ is abelian, and $\mathbf{R}(G)=\operatorname{NKer}_{\mathbb{R}}(G)=\left\langle g^{2}\right\rangle$ otherwise.

Proof. First, observe that $g^{2}=\left(g^{2}\right)^{g}=g^{-2}$ and thus $g^{4}=1$. Moreover, for any $a \in A$, we have $(g a)^{2}=g^{2} a^{g} a=g^{2}$. By assumption, $g^{2} \neq 1$.

In view of Lemma 5.1, it suffices to show that $\left\langle g^{2}\right\rangle \subseteq \operatorname{NKer}_{\mathbb{R}}(G)$, that is, all characters $\chi \in \operatorname{Irr} G$ with $g^{2} \notin \operatorname{Ker} \chi$ are skew-linear. (In the case when $G /\left\langle g^{2}\right\rangle$ is abelian, all characters of $G /\left\langle g^{2}\right\rangle$ are linear and thus it will follow that all characters of $G$ are skew-linear and $G$ is Dedekind. Conversely, if $\operatorname{NKer}_{\mathbb{R}}(G)>\left\langle g^{2}\right\rangle$, then $\operatorname{NKer}_{\mathbb{R}}(G)=G$ by Lemma 5.1, and then $G \cong Q_{8} \times\left(C_{2}\right)^{r}$ by Theorem 4.6 and $G /\left\langle g^{2}\right\rangle$ is abelian.)

So suppose that $\chi \in \operatorname{Irr} G$ is not linear, and $g^{2} \notin \operatorname{Ker} \chi$. Let $\lambda \in \operatorname{Lin} A$ be a constituent of the restriction $\chi_{A}$. Then $\chi=\lambda^{G}$ by Clifford theory [10, Corollary 6.19]. As $a^{g}=a^{-1}$ for all $a \in A$, we have $\lambda^{g}=\bar{\lambda}$. Also, $\lambda\left(g^{2}\right) \neq 1$, and thus $\lambda\left(g^{2}\right)=-1$ and $\chi\left(g^{2}\right)=-2$. It follows that

$$
\begin{aligned}
\nu_{2}(\chi)=\frac{1}{|G|} \sum_{x \in G} \chi\left(x^{2}\right) & =\frac{1}{|G|} \sum_{a \in A}\left(\chi\left((g a)^{2}\right)+\chi\left(a^{2}\right)\right) \\
& =\frac{1}{|G|}\left(-2|A|+\sum_{a \in A}\left(\lambda\left(a^{2}\right)+\overline{\lambda\left(a^{2}\right)}\right)\right) \\
& =\frac{-2|A|}{|G|}=-1 .
\end{aligned}
$$

Here we have used that $(g a)^{2}=g^{2}$ for all $a \in A$, and that $\sum_{a \in A} \lambda\left(a^{2}\right)=$ $\sum_{a \in A} \lambda^{2}(a)=0$ since $\bar{\lambda} \neq \lambda$ and thus $\lambda^{2} \neq 1$. Since $\nu_{2}(\chi)=-1$ and $\chi(1)=2$, it follows that $\chi$ is indeed skew-linear, as claimed.

5.3. Lemma. When $G=\langle u\rangle \times\langle x, y\rangle \times E$ with $\langle u\rangle \cong C_{4},\langle x, y\rangle \cong Q_{8}$ and $E \cong\left(C_{2}\right)^{r}$, then $\operatorname{NKer}_{\mathbb{R}}(G)=\mathbf{R}(G)=\left\langle u^{2} x^{2}\right\rangle \neq 1$.

Proof. As $\langle u x\rangle \not G$, we have $\mathbf{R}(G) \leqslant\left\langle u^{2} x^{2}\right\rangle$. Let $\tau$ be the nonlinear irreducible character of $\langle x, y\rangle$ and $\lambda$ a character of $\langle u\rangle$ with $\lambda \neq \bar{\lambda}$. If $\chi$ is a character with $\chi\left(u^{2} x^{2}\right) \neq \chi(1)$, then either $\chi$ is linear, or $\chi=\lambda^{2} \times \tau \times \sigma$ for some $\sigma \in \operatorname{Lin} E$. The latter characters all have $\nu_{2}(\chi)=-1$. Thus $\left\langle u^{2} x^{2}\right\rangle \subseteq \operatorname{NKer}_{\mathbb{R}}(G)$.

5.4. Lemma. When $G=\langle u, v\rangle \times\langle x, y\rangle \times E$ with $\langle u, v\rangle \cong\langle x, y\rangle \cong Q_{8}$ and $E \cong\left(C_{2}\right)^{r}$, then $\operatorname{NKer}_{\mathbb{R}}(G)=\mathbf{R}(G)=\left\langle u^{2} x^{2}\right\rangle \neq 1$.

Proof. As $\langle u x\rangle \not G$, we have $\mathbf{R}(G) \leqslant\left\langle u^{2} x^{2}\right\rangle$. Let $\tau_{1}$ and $\tau_{2}$ be the nonlinear characters of $\langle u, v\rangle$ and $\langle x, y\rangle$, respectively. If $\chi\left(u^{2} x^{2}\right) \neq \chi(1)$, then either $\chi=$ 
$\tau_{1} \times \lambda \times \sigma$ with $\lambda \in \operatorname{Lin}\langle x, y\rangle$ and $\sigma \in \operatorname{Lin}(E)$, or $\chi=\lambda \times \tau_{2} \times \sigma$ with $\lambda \in \operatorname{Lin}\langle u, v\rangle$ and $\sigma \in \operatorname{Lin}(E)$. In both cases, $\nu_{2}(\chi)=-1$ and thus $\left\langle u^{2} x^{2}\right\rangle \leqslant \operatorname{NKer}_{\mathbb{R}}(G)$.

This lemma finishes the proof of the "if" part of Theorem B. We now start working for the "only if"-part.

5.5. Lemma. Suppose that $1<\operatorname{NKer}_{\mathbb{R}}(G)<G$ and that $G$ is not a 2 -group. Then $G$ is generalized dicyclic.

Proof. By Corollary 4.3 and Lemma 5.1, we have that $\operatorname{NKer}_{\mathbb{R}}(G)=\mathbf{R}(G)=\langle z\rangle$, where $z$ has order 2 . Every odd-order subgroup of $G$ is normal in $G$, and in particular the Sylow $p$-subgroups, for $p$ odd, generate a normal 2-complement, $U$, of $G$. As $U$ is Dedekind, it follows that $U$ is abelian.

Now set $A=\mathbf{C}_{G}(U)$, which contains $U$. There is $g \in G$ such that $\langle g\rangle \not G$. By Lemma 5.1, we have $g^{4}=1$. If $g u=u g$ for some $u \in U$, then $\langle g\rangle$ is characteristic in $\langle g u\rangle=\langle g\rangle \times\langle u\rangle$, and thus $\langle g u\rangle \not G$. Again by Lemma 5.1, it follows that $(g u)^{4}=1$ and thus $u=1$. Thus $\mathbf{C}_{U}(g)=1$ and $g \notin A$. In particular, $A<G$.

Conversely, let $g \notin A$, and let $s=g_{2}$ be the 2-part of $g$. Then $g A=s A$ and thus $s \notin A$. Thus $u^{s} \neq u$ for some $u \in U$, and thus $s^{u}=s[s, u] \notin\langle s\rangle$. It follows that $\langle s\rangle$ is not normal in $G$, and thus $\langle g\rangle$ is not normal in $G$. By Lemma 5.1, it follows that $g^{4}=1$ and $g^{2}=z$ (and thus $s=g$ ).

In particular, for $g \in G \backslash A$ and $a \in A$, we have $g^{2}=z=(g a)^{2}=g^{2} a^{g} a$, and thus $a^{g}=a^{-1}$. For $u \in U$ and $g, h \in G \backslash A$ we have $u^{g}=u^{-1}=u^{h}$ and thus $g h^{-1} \in \mathbf{C}_{G}(U)=A$, so $|G: A|=2$. Thus $G$ is generalized dicyclic.

To finish the proof of the "only if" part of Theorem B, we use a part of Blackburn's classification [3, Theorem 1]:

5.6. Theorem (Blackburn 1966). Let $G$ be a p-group with $\mathbf{R}(G) \neq 1$. Then one of the following holds:

(i) $G$ is abelian.

(ii) $p=2$ and $G$ is generalized dicyclic.

(iii) $p=2$ and $G \cong C_{4} \times Q_{8} \times\left(C_{2}\right)^{r}, r \in \mathbb{N}$.

(iv) $p=2$ and $G \cong Q_{8} \times Q_{8} \times\left(C_{2}\right)^{r}, r \in \mathbb{N}$.

Using Theorem 5.6, it is rather straightforward to determine all finite groups $G$ with $\mathbf{R}(G) \neq 1$, but a rather long list emerges [3, Theorem 2]. However, due to Lemma 5.5, we do not need to go through the longer list of finite groups with $\mathbf{R}(G) \neq 1$.

Proof of Theorem B, "only if". Suppose that $\operatorname{NKer}_{\mathbb{R}}(G) \neq 1$. If $\operatorname{NKer}_{\mathbb{R}}(G)=G$, then $G$ is abelian or $G \cong Q_{8} \times\left(C_{2}\right)^{r}$, by Theorem 4.6.

If $1<\operatorname{NKer}_{\mathbb{R}}(G)<G$ and $G$ is not a 2-group, then $G$ is generalized dicyclic, by Lemma 5.5. If $G$ is a 2-group, then it follows from Blackburn's classification of 2groups with $\mathbf{R}(G) \neq 1$ (Theorem 5.6) that $G$ appears on the list in Theorem B. 


\section{Clasification OVer the RAtional Numbers}

In this section, we prove Theorem D. Throughout, we write $\operatorname{NKer}(G):=$ $\operatorname{NKer}_{\mathbb{Q}}(G)$.

First, we show the "if"-part of Theorem D. We collect the necessary information in the following theorem:

6.1. Theorem. Let $G$ be a finite group.

(i) When $G$ is abelian, then $\operatorname{NKer}(G)=G$.

(ii) Suppose $G=S \times A$, where $S$ is a 2-group of exponent 4 which appears on the list from Theorem $B$, the group $A$ is abelian of odd order, and the multiplicative order of 2 modulo $|A|$ is odd. Then $1 \neq \operatorname{NKer}_{\mathbb{R}}(S) \leqslant$ $\operatorname{NKer}(G)$.

(iii) When $G$ is generalized dicyclic, then $\{1\} \neq \operatorname{NKer}_{\mathbb{R}}(G) \leqslant \operatorname{NKer}_{\mathbb{Q}}(G)$.

(iv) Let $G=(P Q) \times B$ be as in Theorem $D$ (iv). Then $1 \neq \operatorname{NKer}(G) \leqslant\left\langle g^{p^{c}}\right\rangle<$ $\langle g\rangle$, with $g \in P$ and $c \geqslant 1$ as in Lemma $C$.

(v) Let $G=Q_{8} \times\left(C_{2}\right)^{r} \times H$ with $H$ a group of odd order, such that the multiplicative order of 2 in $(\mathbb{Z} /|H|)^{*}$ is odd. Then $\operatorname{NKer}(H) \leqslant \operatorname{NKer}(G)$.

We remark that in Case (iii), we actually have $\operatorname{NKer}_{\mathbb{R}}(G)=\operatorname{NKer}(G)$, and in Case (ii), we have $\operatorname{NKer}(G)=G$ when $\operatorname{NKer}_{\mathbb{R}}(S)=S$, and $\operatorname{NKer}(G)=\operatorname{NKer}_{\mathbb{R}}(S)$ else. This is not too difficult to see, but as we do not need this, we omit the proofs. In the situation of Theorem 6.1(v), it follows from Lemma 3.4 that $\operatorname{NKer}(G)=\operatorname{NKer}(H)$ whenever $\operatorname{NKer}(H)<H$. In the situation of (iv), suppose that $s \geqslant c$ is such that the factor group $G /\left\langle g^{p^{s}}\right\rangle$ still fulfills the various conditions in Theorem D (iv). Then we must have $g^{p^{s-1}} \in \operatorname{NKer}(G)$ by (iv) above. In fact, one can show that $\operatorname{NKer}(G)=\left\langle g^{p^{s-1}}\right\rangle$, where $s$ is the smallest number such that $G /\left\langle g^{p^{s}}\right\rangle$ is as in Theorem D (iv).

Notice that Theorem 6.1 (iv) contains Lemma C from the introduction. Theorem 6.1 shows in particular that $\operatorname{NKer}(G) \neq 1$ for all the groups occurring in Theorem D.

Proof of Theorem 6.1, (i)-(iii) and (v). Part (i) is trivial, and (iii) follows from Lemma 5.2 and Lemma 3.1.

Now assume the situation of (ii). By Theorem B, we have $\operatorname{NKer}_{\mathbb{R}}(S) \neq 1$. Pick $\chi \in \operatorname{Irr}(G)$ with $\operatorname{NKer}_{\mathbb{R}}(S) \nsubseteq \operatorname{Ker}(\chi)$. We have to show that $\chi$ is skew-linear over $\mathbb{Q}$. We can write $\chi=\tau \times \lambda$ with $\tau \in \operatorname{Irr}(S)$ and $\lambda \in \operatorname{Lin}(A)$. Since $\operatorname{NKer}_{\mathbb{R}}(S) \nsubseteq \mathbb{K e r}(\tau)$, we either have $\tau(1)=1$, or $\tau(1)=2$ and $\nu_{2}(\tau)=-1$. When $\tau(1)=1$, then $\chi$ is linear. In the second case, $\mathbb{R} S e_{\tau}$ is isomorphic to the quaternions over the reals. Since $S$ has exponent 4 , we have that $\mathbb{Q}(\tau)=\mathbb{Q}$ and $\mathbb{Q} S e_{\tau} \cong \mathbb{H}$, the rational quaternions. Thus $\mathbb{Q} G e_{\chi} \cong \mathbb{H} \otimes_{\mathbb{Q}} \mathbb{Q}(\lambda)$. By assumption and by Lemma 4.4(iii), the latter is a division ring. Thus $\chi$ is skew-linear over $\mathbb{Q}$ as required. 
Finally, let $G$ be as in (v), and write $S=Q_{8} \times\left(C_{2}\right)^{r}$. Let $\chi \in \operatorname{Irr}(G)$ be such that $\operatorname{NKer}(H) \nsubseteq \operatorname{Ker}(\chi)$. We have to show that $\chi$ is skew-linear over $\mathbb{Q}$. We can write $\chi=\sigma \times \vartheta$ with $\sigma \in \operatorname{Irr}(S)$ and $\vartheta \in \operatorname{Irr}(H)$. As $\operatorname{NKer}(H) \nsubseteq \operatorname{Ker}(\vartheta)$, the character $\vartheta$ is skew-linear over $\mathbb{Q}$. Let $D$ be the block ideal of $\mathbb{Q} H$ corresponding to $\vartheta$. This is a division ring with center isomorphic to $\mathbb{Q}(\vartheta)$. If $\sigma$ is linear, then the block ideal corresponding to $\chi=\sigma \times \vartheta$ is again isomorphic to $D$. If $\sigma$ is nonlinear, then the block ideal corresponding to $\chi$ is isomorphic to

$$
\left(\mathbb{H} \otimes_{\mathbb{Q}} \mathbb{Q}(\vartheta)\right) \otimes_{\mathbb{Q}(\vartheta)} D,
$$

by Lemma 2.4. The first factor is a division ring by Lemma 4.4(iii). Therefore, the tensor product is also a division ring, since both factors are division rings, and the first has dimension 4 over its center, while $D$ has odd dimension $\vartheta(1)^{2}$ over its center. Thus $\chi$ is skew-linear. This shows that $\operatorname{NKer}(H) \leqslant \operatorname{NKer}(G)$ as required.

For the proof of Theorem 6.1 (iv), we need some lemmas first. Recall that $m_{q}(\chi)$ denotes the Schur index over $\mathbb{Q}_{q}$, the field of $q$-adic numbers.

6.2. Lemma. Let $G$ be a finite group and $q$ a prime. Suppose that $G$ has a normal abelian Sylow q-subgroup $Q$, such that every subgroup of $Q$ is normal in $G$. Let $H$ be a q-complement in $G$. If $\chi \in \operatorname{Irr}(G)$, then $m_{q}(\chi)=\left|\mathbb{Q}_{q}(\chi, \vartheta): \mathbb{Q}_{q}(\chi)\right|$ for any irreducible constituent $\vartheta \in \operatorname{Irr}(H)$ of $\chi_{H}$.

Notice that the $q$-complement $H$ exists by the Schur-Zassenhaus theorem. We allow $Q=1$ and $H=G$ in this lemma.

Proof of Lemma 6.2. Let $\lambda \in \operatorname{Lin}(Q)$ be a constituent of $\chi_{Q}$. Then $K=\operatorname{Ker}(\lambda)$ is normal in $G$ by assumption and thus $K \subseteq \operatorname{Ker}(\chi)$. We may factor out $K$ and assume without loss of generality that $K=1$.

This means that $Q$ is cyclic and thus $\chi$ is in a $q$-block with cyclic defect group. Thus we can apply Benard's theorem [2] to $\chi$ and conclude that $m_{q}(\chi)=$ $\left|\mathbb{Q}_{q}(\chi, \varphi): \mathbb{Q}_{q}(\chi)\right|$ for any irreducible Brauer constituent $\varphi$ of $\chi$. But an irreducible Brauer character of $G$ contains the normal $q$-subgroup $Q$ in its kernel, and thus can be identified with an ordinary character of the $q^{\prime}$-group $H \cong G / Q$. Thus if $\varphi$ is an irreducible Brauer constituent of $\chi$, then $\varphi_{H}=\vartheta \in \operatorname{Irr}(H)$ is an irreducible constituent of $\chi$, and the result follows from Benard's theorem.

The next observation was already used in Blackburn's classification.

6.3. Lemma. Let $Q$ be a finite abelian q-group (q prime) and let $P$ be some finite group with $(|P|,|Q|)=1$. Suppose that $P$ acts on $Q$ by automorphisms such that every subgroup of $Q$ is $P$-invariant. Then $P / \mathbf{C}_{P}(Q)$ is cyclic of order dividing $q-1$, and $\mathbf{C}_{P}(x)=\mathbf{C}_{P}(Q)=P_{\lambda}$ for every $1 \neq x \in Q$ and $1_{Q} \neq \lambda \in \operatorname{Lin} Q$. 
Proof. Take $x \in Q$ of maximal order and $u \in P$. Since $x^{u} \in\langle x\rangle$ by assumption, we have $x^{u}=x^{k}$ for some $k \in \mathbb{N}$. If $y \in Q$ with $\langle x\rangle \cap\langle y\rangle=1$, then $y^{u}=y^{k}$, since $u$ maps $\langle y\rangle$ and $\langle x y\rangle$ to itself. It follows that $y^{u}=y^{k}$ for all $y \in Q$.

Therefore, $P / \mathbf{C}_{P}(Q)$ is isomorphic to a $q^{\prime}$-subgroup of Aut $(\langle x\rangle)$, and thus is cyclic of order dividing $q-1$.

Finally, suppose $1 \neq x \in Q$ and $x^{u}=x$ for some $u \in P$. As we have just seen, there is $k \in \mathbb{N}$ such that $y^{u}=y^{k}$ for all $y \in Q$. It follows that $k \equiv 1 \bmod q$ (as $q \mid \operatorname{ord}(x)$ ). Since $\left|P / \mathbf{C}_{P}(Q)\right|$ divides $q-1$, it follows that $k^{q-1} \equiv 1 \bmod q^{n}$, where $q^{n}$ is the exponent of $Q$. But this yields that $k \equiv 1 \bmod q^{n}$ and thus $u \in \mathbf{C}_{P}(Q)$ as claimed. The proof for $\lambda \in \operatorname{Lin} Q$ is similar, using that there is $\ell$ such that $\mu^{u}=\mu^{\ell}$ for all $\mu \in \operatorname{Lin} Q$.

Next, we compute some $q$-adic Schur indices for a class of groups containing those in Theorem D (iv). This result will also be used later in the proof of the "only if"-part.

6.4. Lemma. Let $p \neq q$ be primes, let $P$ be an abelian $p$-group and $Q$ an abelian $q$-group. Suppose $P$ acts on $Q$ by automorphisms such that every subgroup of $Q$ is $P$-invariant, and set $C=\mathbf{C}_{P}(Q)$. Let $B$ be an abelian group with $(p q,|B|)=1$ and let $G=(P Q) \times B$. Then any nonlinear $\chi \in \operatorname{Irr}(G)$ has the form $\chi=(\mu \times \lambda)^{G}$ for some $\mu \in \operatorname{Lin}(C B)$ and $1 \neq \lambda \in \operatorname{Lin}(Q)$. Let $\vartheta \in \operatorname{Lin}(P B \mid \mu)$. Then $m_{q}(\chi)=\ell / k$, where $\ell$ is the smallest positive integer such that $\operatorname{ord}(\vartheta)$ divides $q^{\ell}-1$, and $k$ is the smallest positive integer such that ord $(\mu)$ divides $q^{k}-1$.

(In other words, $\ell$ and $k$ are the multiplicative orders of $q$ modulo ord $(\vartheta)$ and modulo $\operatorname{ord}(\mu)$, respectively.)

Proof of Lemma 6.4. Notice that $C B \leqslant \mathbf{Z}(G)$, and that $H=P B$ is an abelian $q$-complement of $G$. We are in the situation of Lemma 6.2. Let $\chi \in \operatorname{Irr}(G)$. If $Q \leqslant \operatorname{Ker}(\chi)$, then $\chi$ is linear, since $G / Q=P \times B$ is abelian. (In fact, $Q=G^{\prime}$ when $C<P$.) Otherwise, let $\lambda \neq 1$ be a linear constituent of $\chi_{Q}$. By Lemma 6.3, we have $G_{\lambda}=C B Q$. By Clifford theory, $\chi$ is induced from some linear character of the abelian group $C B Q$, say $\chi=(\mu \times \lambda)^{G}$ with $\mu \in \operatorname{Lin}(C B)$. It follows that $\mathbb{Q}_{q}(\chi)=\mathbb{K}(\mu)$, where $\mathbb{K} \subseteq \mathbb{Q}_{q}(\lambda)$ is totally ramified over $\mathbb{Q}_{q}$, and the extension $\mathbb{K}(\mu) / \mathbb{K}$ is unramified. By the general form of unramified extensions, the residue field of $\mathbb{Q}_{q}(\chi)=\mathbb{K}(\mu)$ has order $q^{k}$, where $k$ is the smallest positive integer such that $\operatorname{ord}(\mu)$ divides $q^{k}-1$.

The restriction $\chi_{H}$ to the $q$-complement $H=P B$ is the sum of all linear characters $\vartheta \in \operatorname{Lin}(H)$ lying over $\mu$. Thus $\mathbb{Q}_{q}(\chi, \vartheta)=\mathbb{K}(\vartheta)$ is generated by $\mathbb{Q}_{q}(\chi)$ and a root of unity of order $\operatorname{ord}(\vartheta)$. Since $\operatorname{ord}(\vartheta)$ is not divisible by $q$, the extensions $\mathbb{Q}_{q}(\chi, \vartheta) / \mathbb{Q}_{q}(\chi)$ and $\mathbb{K}(\vartheta) / \mathbb{K}$ are unramified. We can thus compute $\left|\mathbb{Q}_{q}(\chi, \vartheta): \mathbb{Q}_{q}(\chi)\right|$ by computing the degrees of the residue fields. As above, the residue field of $\mathbb{Q}_{q}(\chi, \vartheta)=\mathbb{K}(\vartheta)$ has order $q^{\ell}$, where $\ell$ is the smallest positive integer such that ord $\vartheta$ divides $q^{\ell}-1$. Now the result follows from Lemma 6.2. 
6.5. Lemma. Let $G=(P Q) \times B$ be as in Theorem $D$ (iv). Then $\operatorname{NKer}_{\mathbb{Q}_{q}}(G) \cap\langle g\rangle \neq$ 1, with $g \in P$ as in Lemma $C$.

Proof. Recall that $P=\langle g\rangle \times P_{0}$, where $g$ has order $p^{c+d}$ and $C:=\mathbf{C}_{P}(Q)=$ $\left\langle g^{p^{c}}\right\rangle \times P_{0}$. Let $z \in\left\langle g^{p^{c}}\right\rangle$ be an element of order $p$. We claim that $z \in \operatorname{NKer}_{\mathbb{Q}_{q}}(G)$.

Suppose that $\chi(z) \neq \chi(1)$ for $\chi \in \operatorname{Irr} G$. If $\chi(1)>1$, then $\chi=(\mu \times \lambda)^{G}$ as in Lemma 6.4, with $\mu \in \operatorname{Lin}(C B)$ and $1 \neq \lambda \in \operatorname{Lin} Q$.

To compute the Schur index of such a $\chi$, we apply Lemma 6.4. Since $\mu(z) \neq 1$, it follows that $\operatorname{ord}(\mu)=p^{d} n$, where $n$ divides $|B|$. For $\vartheta \in \operatorname{Lin}(P B \mid \mu)$, we have $\operatorname{ord}(\vartheta)=p^{c} \operatorname{ord}(\mu)=p^{c+d} n$. Let $k$ and $\ell$ be the multiplicative order of $q$ modulo $p^{d} n$ and $p^{c+d} n$, respectively. We have to show that $\ell / k=p^{c}$.

Let $p^{e}=(q-1)_{p}$ and write $q=1+p^{e} x$ with $(p, x)=1$. Then $q^{p} \equiv 1+p^{e+1} x \not \equiv 1$ $\bmod p^{e+2}$ except when $p^{e}=2$. By the assumptions in Lemma C, $p \leqslant p^{c} \leqslant p^{e} \leqslant p^{d}$. It follows that $\left(q^{k_{0}}-1\right)_{p}=p^{d}$ for $k_{0}=p^{d-e}$ when $p^{e}>2$. When $p^{e}=2$, the extra condition in Lemma $\mathrm{C}$ also ensures that $\left(q^{k_{0}}-1\right)_{p}=p^{d}$, when $k_{0}$ is the multiplicative order of $q$ modulo $p^{d}$. Additionally, the assumption in Theorem D (iv) on $|B|$ yields that $k / k_{0}$ is not divisible by $p$. Thus $q^{k}=1+p^{d} x$ for some $x \in \mathbb{Z}$ with $(p, x)=1$, and $p^{d}>2$. It follows $\ell=p^{c} k$ and thus $m_{q}(\chi)=p^{c}=\chi(1)$. This was to be shown.

Proof of Theorem 6.1 (iv). As $\operatorname{NKer}_{\mathbb{Q}_{q}}(G) \leqslant \operatorname{NKer}(G)$ for any group, we see that $\operatorname{NKer}(G) \cap\langle g\rangle \neq 1$. On the other hand, we have that $\mathbf{R}(G) \leqslant\left\langle g^{p^{c}}\right\rangle$ (because $\left\langle g^{p^{c-1}}\right\rangle$ is not normal in $\left.P Q\right)$, and thus $\operatorname{NKer}(G) \leqslant\left\langle g^{p^{c}}\right\rangle$ by Lemma 3.7.

This finishes the proof of the "if"-part of Theorem D.

Next, we prove Theorem D for nilpotent groups.

6.6. Theorem. Let $G$ be nilpotent. Then $\operatorname{NKer}(G) \neq 1$ if and only if one of the following holds.

(i) $G$ is abelian and $G \neq\{1\}$.

(ii) $G=S \times A$, where $S \in \operatorname{Syl}_{2}(G)$ is a nonabelian group from the list in Theorem 5.6 and has exponent 4 , and $A$ is abelian of odd order, and the multiplicative order of 2 in $(\mathbb{Z} /|A|)^{*}$ is odd.

(iii) $G$ is a generalized dicyclic 2-group.

Proof. The "if"-part has been shown in Theorem 6.1, so assume $\operatorname{NKer}(G) \neq 1$. Then $1 \neq \operatorname{NKer}(P) \leqslant \mathbf{R}(P)$ for every nontrivial subgroup $P \leqslant G$. By Theorem 5.6, the Sylow subgroups of odd order are all abelian. Thus we have $G=S \times A$ with $S \in \operatorname{Syl}_{2}(G)$ and $A$ abelian of odd order, and $S$ appears on the list from Theorem 5.6. We may assume that $S$ is nonabelian.

If $S$ has exponent 4, then the nonlinear, but skew-linear characters of $S$ yield the quaternions over $\mathbb{Q}$ as block ideal of the rational group algebra $\mathbb{Q} S$, and it follows from Lemma 4.4(iii) and Lemma 2.4 that (ii) holds. 
If $S$ contains elements of order 8 or greater, then $S$ is generalized dicyclic, and $\operatorname{NKer}(S)<S$ is cyclic of 2 -power order. It remains to show that $A=1$ in this case. Let $z \in \operatorname{NKer}(S)$ be the element of order 2. There is a $\sigma \in \operatorname{Irr} S$ such that $S / \operatorname{Ker}(\sigma)$ is a dicyclic (=generalized quaternion) group of order at least 16, and $z \notin \operatorname{Ker}(\sigma)$. Then $\mathbb{Q}(\sigma)$ contains $\sqrt{2}$. As $S / \operatorname{Ker}(\sigma)$ has a subgroup of order 8 isomorphic to the quaternion group, the block ideal of $\mathbb{Q} S$ corresponding to $\sigma$ is isomorphic to the quaternions over a field containing $\sqrt{2}$.

Let $\lambda \in \operatorname{Lin} A$ and $\chi=\sigma \times \lambda$. The block ideal corresponding to $\chi$ is isomorphic to

$$
\left(\mathbb{H} \otimes_{\mathbb{Q}} \mathbb{Q}(\sigma)\right) \otimes_{\mathbb{Q}(\sigma)} \mathbb{Q}(\sigma, \lambda) \cong \mathbb{H} \otimes \mathbb{Q}(\sigma, \lambda),
$$

by Lemma 2.4. By Lemma 4.4(iv), $\mathbb{H} \otimes \mathbb{Q}(\sqrt{2}, \lambda)$ can be a division ring only when $\lambda=1_{A}$. Since $\lambda \in \operatorname{Lin} A$ was arbitrary, we have $A=1$ as required.

The proof of Theorem D ("only if") for nonnilpotent groups will be divided into a number of lemmas. Recall that a Blackburn group is a finite group $G$ such that $\mathbf{R}(G) \neq 1$. By Lemma 3.7, a group with $\operatorname{NKer}(G) \neq 1$ is a Blackburn group. For later reference, we record the following observation (which is part of the argument used by Blackburn to classify these groups):

6.7. Lemma. Let $G$ be a Blackburn group and $p$ a prime dividing $|\mathbf{R}(G)|$. Then $G$ has a normal p-complement $A$ such that every subgroup of $A$ is normal in $G$. If $A$ is nonabelian, then $G=Q_{8} \times\left(C_{2}\right)^{r} \times H$, where $H$ is a Blackburn group of odd order.

Proof. By definition of $\mathbf{R}(G)$, all the Sylow $q$-subgroups for $q \neq p$ are normal in $G$, and thus generate a normal $p$-complement, $A$. By definition of $\mathbf{R}(G)$, it follows also that every subgroup of $A$ is normal in $G$.

In particular, $A$ is a Dedekind group. If $A$ is nonabelian, then $S \in \operatorname{Syl}_{2}(G)$ is isomorphic to $Q_{8} \times\left(C_{2}\right)^{r}$, by Theorem 4.1. As $S \unlhd G$, there is a 2-complement $H$. Since every subgroup of $S$ is normal in $G$, it is easy to see that $H$ centralizes $S$ and thus $G=S \times H$ (this is also shown in [3, Proof of Theorem 2(e)]). Any nonnormal subgroup of $H$ is nonnormal in $G$ and thus $1 \neq \mathbf{R}(G) \leqslant \mathbf{R}(H)$.

Thus $A$ is a Dedekind group and $G=P A$ for any $P \in \operatorname{Syl}_{p}(G)$. The classification of Blackburn groups can now be obtained by considering the different possibilities for $P$ and $A$ (using the fact that $P$ is also a Blackburn group and Theorem 5.6 for $P$, and Theorem 4.1 for $A$ ). However, in our proof of Theorem $\mathrm{D}$, we do not have to consider all the cases of Blackburn's classification separately. The next result will be used to reduce to the case that $A$ is abelian.

6.8. Lemma. Let $G=Q_{8} \times\left(C_{2}\right)^{r} \times H$ with $H \neq 1$ of odd order, and suppose $\operatorname{NKer}(G) \neq 1$. Then $\operatorname{NKer}(H) \neq 1$ and the multiplicative order of 2 in $(\mathbb{Z} /|H|)^{*}$ is odd. 
Proof. When $\operatorname{NKer}(H)=H$ then $H$ is abelian and $G$ is a Dedekind group, and Corollary 4.3 and Theorem 4.5 yield the result. When $\operatorname{NKer}(H)<H$, then $1 \neq \operatorname{NKer}(G) \leqslant \operatorname{NKer}(H)$ by Lemma 3.4 .

Let $z \in \operatorname{NKer}(G)$ have prime order $p$, and let $A$ be the abelian $p$-complement of $H$. Then $\langle z\rangle \unlhd G$ and so $\langle z, A\rangle=\langle z\rangle \times A$. Suppose $\lambda \in \operatorname{Lin}(\langle z, A\rangle)$ has maximal possible order. (This implies $\lambda(z) \neq 1$, in particular.) Then any $\chi \in \operatorname{Irr}(H \mid \lambda)$ is skew-linear. For $\tau \in \operatorname{Irr}\left(Q_{8}\right)$ with $\tau(1)=2$, we must have that $\tau \times \chi$ is also skew-linear. Lemma 2.4 yields in particular, that $\mathbb{Q}(\chi)$ must not be a splitting field for $\mathbb{H}$ (the quaternions over $\mathbb{Q}$ ).

Cleary, $\mathbb{Q}(\chi), \mathbb{Q}(\lambda) \subseteq \mathbb{Q}(\varepsilon)$, where $\varepsilon$ is a primitive $|H|$-th root of unity. Since every prime dividing $|H|$ also divides ord $(\lambda)$, we see that $|\mathbb{Q}(\varepsilon): \mathbb{Q}(\lambda)|$ is odd. By Lemma 2.2, all constituents of $\chi_{A}$ are Galois conjugate with $\lambda$ over $\mathbb{Q}(\chi)$. Thus $|\mathbb{Q}(\chi, \lambda): \mathbb{Q}(\chi)|$ divides $\chi(1)$ and is odd. It follows that $|\mathbb{Q}(\varepsilon): \mathbb{Q}(\chi)|$ is odd as well. Thus $\mathbb{Q}(\chi)$ is a splitting field for the quaternions, if and only if $\mathbb{Q}(\varepsilon)$ is a splitting field for the quaternions. Now Lemma 4.4(iii) yields that the condition on the order of $2 \bmod |H|$ holds.

6.9. Lemma. Let $G=P A$ be a Blackburn group with normal abelian p-complement $A$ and $\mathbf{R}(G) \leqslant P \in \operatorname{Syl}_{p}(G)$. Suppose that $\chi \in \operatorname{Irr}(G)$ is skew-linear over $\mathbb{Q}_{q}$, where $q$ is a prime dividing $|A|$. Then $P$ centralizes every Sylow $r$-subgroup $R$ of $A$ such that $r \neq q$ and $R \nsubseteq \operatorname{Ker}(\chi)$.

Proof. Let $r \neq q$ and $R \in \operatorname{Syl}_{r}(A)$, and assume that $R \nsubseteq \operatorname{Ker}(\chi)$. Let $\lambda \in \operatorname{Lin}(R)$ be a linear constituent of $\chi_{R}$, so that $\lambda \neq 1$.

By Lemma $6.3, \mathbf{C}_{P}(R)=\mathbf{C}_{P}(x)$ for any $1 \neq x \in R$, and thus also

$$
\mathbf{C}_{P}(R)=P_{\lambda}:=\left\{u \in P \mid \lambda^{u}=\lambda\right\} .
$$

Consider the subgroup $H=P R$, and choose a constituent $\vartheta \in \operatorname{Irr}(H)$ of $\chi_{H}$ that lies over $\lambda$. Then $\vartheta=\psi^{H}$ for some $\psi \in \operatorname{Irr}\left(H_{\lambda}\right)$, where $H_{\lambda}=\mathbf{C}_{P}(R) R$. Thus $\vartheta(1) \geqslant\left|P: \mathbf{C}_{P}(R)\right|$. On the other hand, by Corollary 2.6 we have that $\vartheta(1)=1$, and thus $P=\mathbf{C}_{P}(R)$ as claimed.

6.10. Lemma. Let $G=P A$ be a finite group, where $A$ is a normal abelian p-complement and $1 \neq \operatorname{NKer}(G) \leqslant P \in \operatorname{Syl}_{p}(G)$. Suppose that $\left|P: \mathbf{C}_{P}(A)\right|>2$. Then $P$ is abelian, and there is exactly one Sylow subgroup of $A$ which is not centralized by $P$.

Proof. Let $z \in \operatorname{NKer}(G) \subseteq P$ be an element of order $p$. Choose $\tau \in \operatorname{Irr}(P)$ with $z \notin \operatorname{Ker}(\tau)$. For $\lambda \in \operatorname{Lin}(A)$ arbitrary, we have

$$
\left[\left(\tau^{G}\right)_{A}, \lambda\right]_{A}=\left[\left(\tau_{P \cap A}\right)^{A}, \lambda\right]_{A}=\left[\tau_{P \cap A}, \lambda_{P \cap A}\right]_{P \cap A}=\tau(1)>0,
$$

as $P \cap A=1$. Thus for any $\lambda \in \operatorname{Lin}(A)$, there is $\chi \in \operatorname{Irr}(G)$ such that $\left[\chi, \tau^{G}\right]>0$ and $\left[\chi_{A}, \lambda\right]>0$. We apply this to a $\lambda$ such that $\lambda_{R} \neq 1_{R}$ for each Sylow subgroup, 
$R$, of $A$. Thus there is a $\chi \in \operatorname{Irr}(G)$ lying over $\tau$ and such that $\operatorname{Ker}(\chi)$ contains no Sylow subgroup of $A$.

Notice that $\mathbf{C}_{P}(A)=P_{\lambda}$ for such a $\lambda$, by Lemma 6.3. As $\chi$ is induced from a character of $G_{\lambda}$, it follows that $\chi(1) \geqslant\left|G: G_{\lambda}\right|=\left|P: \mathbf{C}_{P}(A)\right|>2$.

Because $z \notin \operatorname{Ker}(\chi)$ and $z \in \operatorname{NKer}(G)$, it follows that $\chi$ is skew-linear over $\mathbb{Q}$ and thus $m_{\mathbb{Q}}(\chi)=\chi(1)$. By Ito's theorem [10, Theorem 6.15], $\chi(1)$ divides $|G: A|=|P|$ and thus is a power of $p$. It follows from Lemma 2.5(i) that there is a prime $q$ (possibly infinite) such that $m_{q}(\chi)=m_{\mathbb{Q}}(\chi)=\chi(1)$.

Since $\chi(1) \geqslant\left|P: \mathbf{C}_{P}(A)\right|>2$, it follows from Lemma 2.5(ii) that the prime $q$, such that $m_{q}(\chi)=\chi(1)$, must be a finite, odd prime and $q \neq p$. It follows that $q$ divides $|A|$. Now Corollary 2.6 yields that $\tau$ is linear. Since the only assumption on $\tau \in \operatorname{Irr} P$ was that $z \notin \operatorname{Ker}(\tau)$, Lemma 3.2 yields that $P$ is abelian. Lemma 6.9 yields that $P$ centralizes all Sylow subgroups of $A$ except the Sylow $q$-subgroup.

6.11. Lemma. Let $G=S A$ be a finite group, where $A$ is a normal (abelian) 2 -complement and $1 \neq \operatorname{NKer}(G) \leqslant S \in \operatorname{Syl}_{2}(G)$, and suppose $\left|S: \mathbf{C}_{S}(A)\right|=2$. Then either $G$ is as in Lemma 6.10 (with $p=2$ and $P=S$ ), or $G$ is generalized dicyclic.

(Notice that $A$ is automatically abelian here since $A$ is a Dedekind group of odd order.)

Proof of Lemma 6.11. First we show that $C:=\mathbf{C}_{S}(A)$ is abelian. Let $z \in \operatorname{NKer}(G)$ have order 2. Then $z \in \mathbf{Z}(G)$ and thus $z \in C$. If $C$ is not abelian, there is $\tau \in \operatorname{Irr}(C)$ with $\tau(z) \neq \tau(1)>1$ (Lemma 3.2). Let $t \in S \backslash C$, and let $\lambda \in \operatorname{Lin}(A)$ be such that $\lambda^{t} \neq \lambda$. Then $\chi:=(\tau \times \lambda)^{G} \in \operatorname{Irr}(G)$, and $\chi(1)=2 \tau(1)>2$. By Lemma 2.5 (ii), $\chi$ can not be skew-linear over $\mathbb{R}$ or $\mathbb{Q}_{2}$. Since $\chi_{C}$ has a non-linear constituent, $\chi$ can not be skew-linear over $\mathbb{Q}_{q}$ for odd primes $q$, by Corollary 2.6. As $\chi(1)=2^{r}$, it follows from Lemma 2.5 (i) that $m_{\mathbb{Q}}(\chi)<\chi(1)$, and thus $z \notin \operatorname{NKer}(G)$, contradiction. Thus $C$ is abelian as claimed, and $G=S A$ has the abelian subgroup $C A=C \times A$ of index 2 .

Fix $t \in S \backslash C$. Notice that $A=[A, t] \times C_{A}(t)$. Since every subgroup of $A$ is normal in $G$, the factors of this decomposition have coprime orders. Also, we have $[A, t] \neq 1$ by assumption, and $t$ inverts the elements in $[A, t]$.

Consider first the case $\mathbf{C}_{A}(t) \neq 1$. Pick some $\lambda \in \operatorname{Lin}(A)$ such that $\operatorname{Ker}(\lambda)$ contains no Sylow subgroup of $A$. Then $\lambda^{t} \notin\{\lambda, \bar{\lambda}\}$. Consider some $\nu \in \operatorname{Lin}(C)$ with $\nu(z)=-1$, where $z \in \operatorname{NKer}(G)$ has order 2 as before, and set $\mu=\nu \times \lambda \in$ $\operatorname{Lin}(C A)$. As $\mu^{t} \neq \mu$, we have $\chi=\mu^{G} \in \operatorname{Irr}(G)$. This $\chi$ remains irreducible modulo 2 , and thus $m_{2}(\chi)=1$, by Lemma 2.5 (iii). As $\mu^{t} \neq \bar{\mu}$, we have also $m_{\mathbb{R}}(\chi)=1$. But as $z \notin \operatorname{Ker}(\chi)$, it follows that $m_{q}(\chi)=2$ for some odd prime $q$ dividing $|A|$. Then Lemma 6.9 yields that $S$ centralizes every Sylow subgroup of $A$ except one. Also Corollary 2.6 yields that $\chi_{S}$ is a sum of linear characters. It 
follows that $\nu^{t}=\nu$ for all $\nu \in \operatorname{Lin}(C)$ with $\nu(z)=-1$. Thus $S$ is abelian and $G$ is as in Lemma 6.10 with $p=2$ in this case.

Now assume that $\mathbf{C}_{A}(t)=1$. If $S$ is abelian and $C$ is not just an elementary abelian 2-group, then again we find $\mu \in \operatorname{Lin}(C A)$ with $\mu^{t} \notin\{\mu, \bar{\mu}\}$ and $\chi=\mu^{G}$ as above, so that $m_{q}(\chi)=2$ for some odd prime $q$, and the result follows again.

If $S$ is abelian and $C$ is elementary 2-abelian, then $G=S[A, t]$ is generalized dicyclic.

Finally, assume that $\mathbf{C}_{A}(t)=1$ and that $S$ is nonabelian. The 2-group $S$ occurs on the list in Theorem 5.6. If $S \cong C_{4} \times Q_{8} \times\left(C_{2}\right)^{r}$ or $S \cong Q_{8} \times Q_{8} \times\left(C_{2}\right)^{r}$, then $S$ is generated by elements $u$ such that $\langle u\rangle \cap \mathbf{R}(S)=1$ and thus $S$ would centralize $A$, so this is impossible. (Alternatively, look at Blackburn's list [3, Theorem 2].) Thus $S$ is generalized dicyclic, and $S$ has an abelian subgroup $D$ of index 2, such that $d^{s}=d^{-1}$ for all $d \in D$ and $s \in S \backslash D$. If $D=C$, then $G$ is generalized dicyclic. Thus we may assume that $D \neq C$. If $S$ is Dedekind, then $S \cong Q_{8} \times\left(C_{2}\right)^{r}$, and we could choose $D=C$. So we can assume that $S$ is not Dedekind, and thus $\mathbf{R}(S)=\langle z\rangle$ by Lemma 5.2. We may choose $t \in D \backslash C$ and $s \in C \backslash D$. Since both $C$ and $D$ are abelian, it follows that $s$ centralizes $C \cap D$, and at the same time inverts the elements in $C \cap D$. Thus $C \cap D$ has exponent 2. Since $|S: C|=|S: D|=2$ and $z \in\langle s\rangle \cap\langle t\rangle$, it follows $s^{2}=t^{2}=z$. Since $s t \notin D$, we also have $(s t)^{2}=z$. It follows that $\langle s, t\rangle \cong Q_{8}$ and $S=\langle s, t\rangle \times(C \cap D) \cong Q_{8} \times\left(C_{2}\right)^{r}$. But then $S$ is Dedekind and $G$ generalized dicyclic, contradiction.

Finally, we recall a part of $[3$, Theorem 2(a)].

6.12. Lemma. Let $G=P A$ be a nonabelian Blackburn group such that $\mathbf{R}(G) \leqslant$ $P \in \operatorname{Syl}_{p}(G)$ and such that $P$ and the normal $p$-complement $A$ are abelian. Then we can write $P=\langle g\rangle \times P_{0}$, such that $\mathbf{C}_{P}(A)=\left\langle g^{p^{c}}\right\rangle \times P_{0}(c \geqslant 1)$, and $p^{d}:=\operatorname{ord}\left(g^{p^{c}}\right)$ is the exponent of $\mathbf{C}_{P}(A)$. There is a $k \in \mathbb{N}$ such that $a^{g}=a^{k}$ for all $a \in A$.

Proof of Theorem D. Assume that $\operatorname{NKer}(G) \neq 1$ and let $p$ be a prime dividing $|\operatorname{NKer}(G)|$. By Lemma 6.7, we have that $G=P A$, where $P \in \operatorname{Syl}_{p}(G)$ and $A$ is a normal $p$-complement and a Dedekind group. By Lemma 6.8, we may assume that $A$ is abelian. By Theorem 6.6, we can assume that $G$ is not nilpotent, and thus $\mathbf{C}_{P}(A)<P$. We assume that $G$ is not generalized dicyclic. By Lemmas 6.10 and 6.11, we can assume that $G=(P Q) \times B$ and $A=Q \times B$, where $Q \in \operatorname{Syl}_{q}(G)$, and that $P$ is also abelian. Thus Lemma 6.12 applies. Write $P=\langle g\rangle \times P_{0}$ and $C=\mathbf{C}_{P}(A)=\left\langle g^{p^{c}}\right\rangle \times P_{0}$, as in that lemma.

Let $z \in \mathbf{R}(G) \subseteq\left\langle g^{p^{c}}\right\rangle$ have order $p$ and notice that we must have $z \in \operatorname{NKer}(G)$, since $1 \neq \operatorname{NKer}(G) \leqslant \mathbf{R}(G)$. Choose $\mu \in \operatorname{Lin}(C B)$ such that $\mu(z) \neq 1$ and $\mu$ has the maximal possible order $p^{d} n$, where $n$ equals the exponent of $B$ and $p^{d}=\operatorname{ord}\left(g^{p^{c}}\right)$. Choose $\lambda \in \operatorname{Lin}(Q), \lambda \neq 1_{Q}$. Then $\chi=(\mu \times \lambda)^{G} \in \operatorname{Irr}(G)$ as in Lemma 6.4. Since $z \notin \operatorname{Ker}(\chi)$, we must have $m_{\mathbb{Q}}(\chi)=\chi(1)$. By the local-global principle (Lemma 2.5 (i)), the Schur index of $\chi$ over some local field must equal 
$\chi(1)=|P: C|=p^{c}$. We claim that this local field must be $\mathbb{Q}_{q}$. If $p^{c}>2$, then we must have $m_{q}(\chi)=\chi(1)$, by Lemma 2.5 (ii) and Lemma 6.9. If $p^{c}=2$, then $\mu \in \operatorname{Lin}(C B)$ has order greater than 2: Namely, when the exponent of $C \times B \leqslant \mathbf{Z}(G)$ divides 2 , then $G$ is generalized dicyclic, and we assume that this is not the case. Thus $m_{\mathbb{R}}(\chi)=1$. Also, $\chi$ is induced from a subgroup of index 2 (which is not a 2 -group), and thus $\chi$ remains irreducible after reducing mod 2 . Thus $m_{2}(\chi)=1$ by Lemma 2.5 (iii). Thus in every case, we must have $m_{q}(\chi)=\chi(1)$.

We can now apply Lemma 6.4. Notice that since $\mu(z) \neq 1$ by assumption, we have $\operatorname{ord}(\vartheta)=p^{c} \operatorname{ord}(\mu)$, as can be seen from the structure of $P$ (Lemma 6.12), and $\operatorname{ord}(\mu)=p^{d} \cdot n$, where $n$ equals the exponent of $B$.

Let $k$ be the order of $q$ in $\mathbb{Z} /\left(p^{d} n\right)$ and $\ell$ the order of $q$ in $\mathbb{Z} /\left(p^{c+d} n\right)$. By Lemma 6.4, we must have $\ell / k=\chi(1)=p^{c}$. This is only possible when $\left(q^{k}-1\right)_{p}=$ $p^{d}>2$. By elementary number theory, the arithmetical conditions in Lemma $\mathrm{C}$ must hold. Moreover, the $p$-part of the multiplicative order of $q$ modulo $n$ must divide the multiplicative order of $q$ modulo $p^{d}$. As $n$ and $|B|$ have the same prime divisors, and $p$ does not divide $|B|$, this means that (iv) in Theorem $\mathrm{D}$ holds.

\section{ACKNOWLEDGMENT}

I wish to thank Erik Friese for a thorough reading of this paper and many useful remarks. I gratefully acknowledge support by the DFG (Project: SCHU 1503/6-1).

\section{REFERENCES}

1. László Babai. Symmetry groups of vertex-transitive polytopes. Geometriae Dedicata 6, no. 3 (1977), pp. 331-337. D OI: 10.1007/BF02429904. MR0486080 (58\#5868), Zbl. 0388.05025 (cit. on p. 2).

2. Mark Benard. Schur indices and cyclic defect groups. Ann. Math. (2) 103, no. 2 (1976), pp. 283-304. D OI: 10.2307/1971007, J S T OR: 1971007. MR0412265, Zbl. 0308. 20012 (cit. on p. 15).

3. Norman Blackburn. Finite groups in which the nonnormal subgroups have nontrivial intersection. J. Algebra 3 (1966), pp. 30-37. D O I: 10.1016/0021-8693 (66) 90018-4. MR0190229, Zbl. 0141.02401 (cit. on pp. 3, 8, 13, 18, 21).

4. Walter Feit. The Representation Theory of Finite Groups. North-Holland Mathematical Library 25. North-Holland, Amsterdam, New York, and Oxford, 1982. MR661045, Zbl. 0493.20007 (cit. on p. 6).

5. Erik Friese. Generic Symmetries of Group Representations. Dissertation. Universität Rostock, 2018. DOI: 10.18453/rosdok_id00002383 (cit. on p. 3).

6. Erik Friese and Frieder Ladisch. Affine symmetries of orbit polytopes. Adv. Math. 288 (2016), pp. 386-425. D OI: 10.1016/j.aim.2015.10.021, arXiv: 1411.0899 [math.MG]. MR3436389, Zbl. 1330.52017 (cit. on p. 2). 
7. Erik Friese and Frieder Ladisch. Classification of affine symmetry groups of orbit polytopes. J. Algebraic Combin. 48, no. 3 (2018), pp. 481-509. D OI: $10.1007 /$ s10801-017-0804-0, arXiv: 1608.06539v4 [math.GR]. MR3864737, Zbl. 06989800 (cit. on p. 2).

8. Bertram Huppert. Endliche Gruppen I. Die Grundlehren der Mathematischen Wissenschaften 134. Springer, Berlin, Heidelberg, and New York, 1967. MR0224703 (37\#302), Zbl. 0217.07201 (cit. on p. 9).

9. Bertram Huppert. Character Theory of Finite Groups. De Gruyter Expositions in Mathematics 25. Walter de Gruyter, Berlin and New York, 1998. D O I: 10.1515/ 9783110809237. MR1645304(99j:20011), Zbl. 0932.20007 (cit. on pp. 3, 4, 10, 11).

10. I. Martin Isaacs. Character Theory of Finite Groups. Dover, New York, 1994. (Corrected reprint of the 1976 edition by Academic Press, New York). MR1280461, Zbl. 0849.20004 (cit. on pp. 3-5, 7, 12, 20).

11. Tsit-Yuen Lam. Introduction to Quadratic Forms Over Fields. Graduate Studies in Mathematics 67. American Mathematical Society, Providence, RI, 2005. MR2104929, Zbl. 1068.11023 (cit. on p. 10).

12. Claude Moser. Représentation de -1 comme somme de carrés dans un corps cyclotomique quelconque. J. Number Theory 5 (1973), pp. 139-141. D o I: 10.1016/ 0022-314x (73) 90067-x. MR0316423, Zbl. 0276.12010 (cit. on p. 10).

13. Irving Reiner. Maximal Orders. L.M.S. Monographs 5. Academic Press, London and New York, 1975. MR0393100 (52\#13910), Zbl. 0305.16001 (cit. on p. 6).

14. Sudarshan K. Sehgal. Nilpotent elements in group rings. Manuscripta Math. 15, no. 1 (1975), pp. 65-80. DOI: 10 . 1007 /bf01168879. MR0364417(51\#671), Zbl. 0302.16010 (cit. on pp. 1, 3, 10).

15. Jean-Pierre Serre. Cours d'arithmétique. Collection SUP: "Le Mathématicien" 2. Presses Universitaires de France, Paris, 1970. MR0255476, Zbl. 0225.12002 (cit. on p. 10).

16. Toshihiko Yamada. The Schur Subgroup of the Brauer Group. Lecture Notes in Mathematics 397. Springer-Verlag, Berlin, 1974. D O I: 10.1007/BFb0061703. MR03 47957 (50\#456), Zbl. 0321.20004 (cit. on p. 6).

Universität Rostock, Institut für Mathematik, 18051 Rostock, GERMANY

E-mail address: frieder.ladisch@uni-rostock.de 